\title{
A new theory of the spin-Peierls transition with special relevance to the experiments on TTFCuBDT
}

\author{
M. C. Cross and Daniel S. Fisher* \\ Bell Laboratories, Murray Hill, New Jersey 07974
}

(Received 8 June 1978)

\begin{abstract}
We develop a new theory of the spin-Peierls transition in spin-1/2 Heisenberg chains, treating the phonons in a mean-field random-phase approximation (RPA) as in previous work, but calculating the relevant response functions of the spin system using the procedure of Luther and Peschel. We show that the RPA on the phonons (and therefore our whole calculation) should be good for the experimentally important system tetrathiafulvalenium bis-cis- $(1,2$ perfluoromethylethylene-1,2-dithiolato)-copper (TTFCuBDT). It is also exact for a model system in which planes of atoms perpendicular to the chains are constrained by lattice forces to move together: we have derived some exact results for the spin-Peierls transition in this model system. We find a new linear dependence of the transition temperature $T_{c}$ on the spin-phonon coupling, and an enhancement of $T_{c}$ and also of the rate of phonon softening above $T_{c}$. Predictions of some other signatures of the transition, such as the specific-heat jump ratio, are however not much changed from previous work. Exact results are found for the leading dependence of the ground-state energy $E$ and gap $\Delta$ in the excitation spectrum on the lattice distortion $\delta: \quad E \propto \delta^{4 / 3}, \Delta \propto \delta^{2 / 3}$
\end{abstract}

\section{INTRODUCTION}

The magnetic phase transition observed at $12{ }^{\circ} \mathrm{K}$ by Jacobs et al,,$^{1,2}$ in tetrathiafulvalenium bis-cis(1,2-perfluoromethylethylene-1,2-dithiolato)-copper (known as TTFCuBDT) was very successfully treated by the authors as a spin-Peierls transition in onedimensional spin- $\frac{1}{2}$ Heisenberg chains. The spin-Peierls transition is most easily understood as the instability of a uniform chain of antiferromagnetically interacting spins towards a dimerized spin-singlet ground state. It is a lattice instability driven by the magnetic interactions. The nomenclature arises from the similarity of the Hamiltonian, when transformed to a pseudofermion representation, to a (spinless) fermion system undergoing the conventional Peierls transition. In one-dimensional systems it is well known that fluctuations are likely to be very important, and for the conventional Peierls case there is a growing understanding of the special importance of a careful treatment of interaction effects in one-dimension. Perhaps the most surprising feature of the experiment was then that these effects did not seem important. Instead the very simple treatment by Pytte ${ }^{3}$ in terms of a mean-field theory of the phonons together with a Hartree approximation on the pseudofermion system was successful in quantitatively accounting for the results.

The situation was apparently complicated by the observations of Moncton et al. ${ }^{4}$ which suggest that the softening of the phonon leading to the phase transition is three-dimensional, and persists to very much higher temperatures than expected; in fact to $220{ }^{\circ} \mathrm{K}$ which should be compared with the transition temperature $T_{c}=12^{\circ} \mathrm{K}$ and with the strength of the magnetic interactions $J=77^{\circ} \mathrm{K}$. We show that the assumption of a three-dimensionally softened phonon pre-existing the magnetic interactions (as suggested by these observations) which is then further softened at low temperatures by the magnetic interactions, allows us to account for the mean-field character of the transition and much of the agreement with simple theories. However, although the phonons may be treated by a mean-field theory, the one-dimensional nature of the spin (pseudofermion) system remains very important, and the Hartree treatment will not be a good approximation. ${ }^{5}$ The theory of the pseudofermions can be markedly improved by using the close similarity of the pseudofermion representation of a one-dimensional Heisenberg chain to the exactly soluble Luttinger-Tomanaga ${ }^{6,7}$ model pointed out by Luther and Peschel. ${ }^{8}$ Using this we predict quite a different dependence of the transition temperature on the spin-phonon coupling constant than the Hartree calculation, and for the particular system under consideration (TTFCuBDT) a reduction in the coupling constant consistent with the known $T_{c}$. We believe that this combined with the pre-existing soft phonon may account for the absence of important phonon fluctuation corrections to the magnetic susceptibility above $T_{c}$, leading to the observed agreement between experiment and the Bonner-Fisher ${ }^{9}$ theoretical predic- 
tions. Our calculations also predict an enhanced softening of the phonon above $T_{c}$. To calculate properties below $T_{c}$ we perform a Ginzburg-Landau expansion. This turns out to have the same form as given by the Hartree approximation, but in terms of an order parameter scaled by a factor $\sqrt{J / T_{c}}$. Thus thermodynamic properties such as the specific-heat jump and susceptibility should be roughly the same as the BCS-like values predicted by Pytte's ${ }^{3}$ calculations, accounting for the rather good agreement with experiment given by this oversimple theory. Nevertheless in comparison with this theory we predict a lattice distortion for a given coupling constant and $T / T_{c}$ reduced by a factor of order $\sqrt{T_{c} / J}$. Finally we calculate new results at absolute zero for the dependence on the lattice distortion parameter $\delta$ of the energy of the spin system for a static lattice distortion $\left(E \propto \delta^{4 / 3}\right)$, and of the gap in the excitation spectrum $\left(\Delta \propto \delta^{2 / 3}\right)$.

Although we write this paper with special reference to the experimentally important TTFCuBDT (and possibly the corresponding gold compound), the new calculations on the spin system are independent of the specific features of this system. Thus we calculate the general response of a one dimensional spin- $\frac{1}{2}$ Heisenberg chain with nearest neighbor interactions to a small lattice distortion assuming only that the dynamics of the lattice may be neglected in this calculation. The calculation of the spin-Peierls transition is then exact for a simple model system of one dimensionally interacting spin chains immersed in a three-dimensional lattice in which the spring constants along the chain direction are much smaller than the spring constants in the planes perpendicular to the chains. These planes then move as a unit and the mean-field random-phase approximation (RPA) treatment of the phonon becomes exact. That the approximation is reasonable for TTFCuBDT follows from the pre-existing soft phonon, which can indeed be thought of as favoring a particular planar motion of the molecules. The relevance of the calculation to other systems (and in particular in calculating $T_{c}$ for a lattice distortion) will depend on the validity of this approximation, possibly resting on quite different arguments.

The plan of the paper is as follows. In Sec. II we introduce the pseudofermion representation of the Hamiltonian and briefly describe the calculation of Pytte that has been used to account for the experimental results. We then describe in Sec. III the special features of the TTFCuBDT system that justify the mean field, random-phase approximation treatment of the phonons. Section IV contains an outline of our treatment of the spin system that takes better account of the one-dimensional nature of the spin interactions. A comparison of the consequences of this treatment with both experiment and the theory of
Pytte is given in Sec. V. Section VI summarizes our understanding of the spin-Peierls transition particularly in TTFCuBDT. A more careful discussion of the mathematical procedures is relegated to the Appendices.

\section{FORMULATION AND THE PYTTE ${ }^{3}$ TREATMENT}

We take the Hamiltonian for the system to be that of a set of noninteracting chains $(\alpha)$ of total $N$ spins ( $\left.\operatorname{spin}=\frac{1}{2}\right)$ where the spins within each chain interact with a nearest-neighbor antiferromagnet Heisenberg interaction

$$
H=\sum_{\alpha l} J(l, l+1 ; \alpha)\left(\overrightarrow{\mathrm{S}}_{l \alpha} \cdot \overrightarrow{\mathrm{S}}_{l+1 \alpha}-\frac{1}{4}\right) .
$$

We assume a linear dependence of $J(l, l+1 ; \alpha)$ on lattice distortion so that in the presence of phonons described by normal coordinates $Q(\overrightarrow{\mathrm{q}})$ the spin-wave coupling is given writing

$$
\begin{aligned}
& J(l, l+1 ; \alpha)= J+\frac{1}{\sqrt{N}} \\
& \times \sum_{q} g(\overrightarrow{\mathrm{q}}) \bullet Q(\overrightarrow{\mathrm{q}}) e^{i \overrightarrow{\mathrm{q}}_{\perp} \cdot \vec{R}_{\alpha}} \\
& \\
& \quad \times e^{i q_{\|} s}\left(1-e^{i \|_{\| l} l^{s}}\right)
\end{aligned}
$$

where $q_{\|}\left(\vec{q}_{1}\right)$ is the component of the phonon wave vector $\overrightarrow{\mathrm{q}}$ parallel (perpendicular) to the chain direction, $\vec{R}_{\alpha}$ is the position of the $\alpha$ th chain, $\mathrm{s}$ is the spacing along the chain, and $g(\vec{q})$ is a coupling constant possibly weakly q dependent. For future use we define an anisotropic $X Y-Z$ Hamiltonian in which the uniform spin coupling is of the form

$$
\begin{aligned}
H_{s}= & J_{\perp} \sum_{l}\left(S_{l}^{x} S_{l+1}^{x}+S_{l}^{y} S_{l+1}^{y}\right) \\
& +J_{z} \sum_{l} S_{l}^{z} S_{l+1}^{z} .
\end{aligned}
$$

It is helpful to transform the spins to a (spinless) pseudofermion representation using the JordanWigner ${ }^{10}$ transformation. As will be seen we can then make use of the growing understanding of onedimensional fermion systems. The transformation on each chain is

$$
S_{l}^{+}=\exp \left(-i \pi \sum_{i}^{l-1} a_{i}^{\dagger} a_{i}\right) a_{l}^{\dagger}=\left(S_{l}^{-}\right)^{\dagger},
$$

where the exponential "counting" factor is needed to give the correct fermion anticommutation rules between operators on different sites. The anticommutation rules then have the usual form within each chain:

$$
\left\{a_{l}^{\dagger}, a_{l^{\prime}}\right\}=\delta_{I^{\prime}}\left\{a_{l}^{\dagger}, a_{l^{\prime}}^{\dagger}\right\}=0=\left\{a_{1}, a_{1^{\prime}}\right\}
$$


Although apparently a rather complicated transformation, for the operators of importance in a spin-Peierls transition the representation is very simple:

$$
S_{l}^{z}=-\frac{1}{2}+a_{l}^{\dagger} a_{l}, \quad S_{l}^{+} S_{l+1}^{-}=a_{l}^{\dagger} a_{l+1}
$$

It is to be noted that the $z$ and $x, y$ components in the spin Hamiltonian are treated in rather different ways. Thus the $x, y$ part leads to a two-fermion ("kinetic energy") term in the pseudofermion Hamiltonian; the $z$ part leads to a four-fermion ("interaction") term. Similarly in the coupling to phonons the $x, y$ part leads to a familiar two-fermion-phonon vertex; the $z$ part to a more complicated four-fermionphonon vertex. It is the treatment of the fourfermion terms that is difficult in this formulation. These terms are just those that distinguish the Heisenberg from the exactly soluble $X Y$ model.

In the absence of phonons the pseudofermion Hamiltonian transformed to $k$ space is (following Pytte $^{3}$ )

$$
H_{f}=H_{0}+H_{\text {int }},
$$

where

$$
H_{0}=\sum_{k} \epsilon_{k} a_{k}^{\dagger} a_{k}
$$

and

$$
H_{\mathrm{int}}=\frac{1}{N} \sum_{k k^{\prime} q} V(q) a_{k+q}^{\dagger} a_{k^{\prime}-q}^{\dagger} a_{k^{\prime}} a_{k}
$$

Here $\epsilon_{k}=J(\cos k a-1), V(q)=J \cos q a$, and

$$
a_{l}^{\dagger}=\frac{1}{\sqrt{N}} \sum_{k} \exp (-i k l s) a_{k}^{\dagger}
$$

For the moment we have specialized the discussion to a single chain, and all wave vectors are along the chain direction; we return to a collection of chains later. In zero magnetic field $\left\langle S_{z}\right\rangle=0$ and hence the free-fermion band is half-filled with Fermi wave vector $k_{F}=\pi / 2 s$. The Jordan-Wigner transformation leading to this Hamiltonian is exact.

The approximation used by Bulaevskii ${ }^{11}$ for the uniform chain, and extended by Pytte $^{3}$ to the coupling to a lattice distortion, is to perform the usual Hartree factorization on the four-fermion terms taking averages in the undistorted state: ${ }^{12}$

$$
\left\langle a_{k}^{\dagger} a_{k^{\prime}}\right\rangle=n_{k} \delta_{k k^{\prime}}
$$

with

$$
n_{k}=\left(e^{\beta E_{k}}+1\right)^{-1}
$$

where $E_{k}=p J \cos k a$ is the one-fermion energy of the approximate Hamiltonian then given:

$$
H_{f}=\sum E_{k} a_{k}^{\dagger} a_{k} \text {. }
$$

The same factorization of the four-fermion-phonon terms leads to the coupling to the phonons

$$
H_{\mathrm{ph}}=\frac{1}{\sqrt{N}} \sum g(k, q) Q(q) a_{k}^{\dagger} a_{k-q},
$$

where $g(k, q)=-i p[\sin k a-\sin (k-q) a] g(q)$. In these expressions

$$
p=1-\frac{2}{N} \sum_{k} n_{k} \cos k a
$$

is a renormalization factor arising from the $z$ spin interactions in this Hartree approximation, and is approximately constant $p=1.64$ at low temperatures $T \ll J$. The approximation thus leads to a Hamiltonian that is the same as for the $X Y$ model, except for a multiplication of the energy scale by the factor p.

The fermion Hamiltonian Eq. (2.9) gives, ${ }^{11}$ with remarkable accuracy, the ground-state energy and low-lying spin-wave excitation frequencies of the uniform Heisenberg chain. The fermion-phonon coupling Eq. (2.10) is reminiscent of the Frohlich Hamiltonian for the Peierls system ${ }^{15}$ : thus, here too, the singularity in the pseudofermion response at $2 k_{F}$ should soften the phonon frequency $\Omega(q)$ at $q=2 k_{F}$ (Kohn anomaly) leading to a phase transition (albeit at zero temperature for an ideally one-dimensional system). For a half-filled band the new phase is the dimerized state.

A random-phase treatment gives for the softening of the phonon

$$
\Omega^{2}(q)=\Omega_{0}^{2}(q)+\Pi(q, \Omega)
$$

where

$$
\Pi(q, \Omega)=\frac{1}{N} \sum_{k}|g(k, q)|^{2} \Pi(k, q, \Omega)
$$

$\Pi(k, q, \Omega)$ is the usual RPA polarizability "bubble"

$$
\Pi(k, q, \Omega)=\left(n_{k}-n_{k-q}\right) /\left(\Omega-E_{k-q}+E_{k}\right) \text {, }
$$

and $\Omega_{0}(q)$ is the phonon frequency in the absence of the spin interactions. At low temperatures the polarizability $\Pi\left(q=2 k_{F}, \Omega=0\right)$ diverges as $\ln J / T$. The transition temperature is given by setting $\Omega$ to zero in Eq. (2.11). Hence the RPA-Hartree calculation predicts a static distortion at $q=2 k_{F}$ below a finite transition temperature $T_{c} \sim J \exp (-1 / \lambda)$, where $\lambda \sim\left|g\left(2 k_{F}\right)\right|^{2} / J \Omega_{0}^{2}\left(2 k_{F}\right)$.

This approach was used rather successfully ${ }^{1,2}$ to explain the first measurements of the phase transition in TTFCuBDT. The results must be seriously doubted, however, since the importance of the onedimensional nature of the system is not fully taken into account. In particular the Hartree factorization underestimates the importance of the four-fermion interaction terms on the divergent $2 k_{F}$ polarizability. In addition, for a one-dimensionally softened pho- 


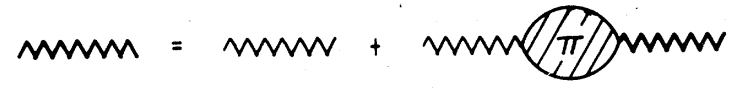

(a)

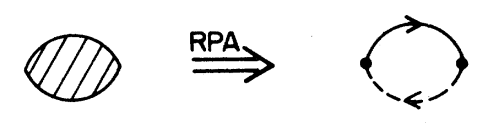

(b)

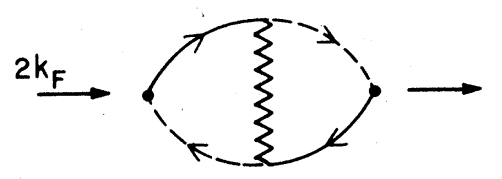

(c)

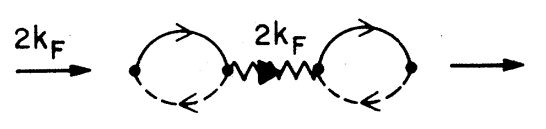

(d)

FIG. 1. (a) Dyson's equation; (b) The random-phase approximation; (c) and (d) two second-order contributions to the expansion of (a). The full (dotted) lines are noninteracting Green's functions for one (two) fermion(s); the light (heavy) zig-zags are noninteracting (exact) phonon Green's functions.

non, fluctuation effects should be important, and the mean-field (random-phase) treatment of the phonons would be inadequate. In the following sections we show that the three-dimensional softening of the phonon suggested by the measurements of Moncton et $a l .{ }^{4}$ in fact justifies the mean-field, random-phase treatment of the phonons. We then proceed to treat the pseudofermions beyond the Hartree approximation to give a much better account of the effect of the interaction terms.

First we must describe the procedure used to immerse the noninteracting spin chains in a threedimensional lattice. The fermion Hamiltonian, Eqs. (2.6) and (2.7), is for a single chain. In the RPA the response of $n$ chains is simply $n$ times the response of one chain, and no further work is required. However to study the validity of RPA it is necessary to look in greater detail at the interaction of a collection of chains with three-dimensional phonons. The slight complication is that (as far as we know) there is no
Jordan-Wigner transformation leading to a pseudofermion representation for three-dimensionally interacting spins: the "fermion" operators defined on different chains in general commute instead of anticommuting. This however leads to no problems providing the interactions do not change the total spin of each chain [which our general Hamiltonian Eq. (2.1) satisfies]. Then for any states $i, j$ of interest

$$
\left\langle i\left|\psi_{i \alpha}^{(+)} \psi_{i}^{(+)}\right| j\right\rangle=0, \quad \alpha \neq \beta,
$$

and within this manifold the anticommutation rules are obeyed. Thus in this somewhat special case, we can map the $n$ chain problem onto a three-

dimensional pseudofermion system, but one in which the bands and interaction depends only on the component of the wave-vector along the chain. For all of our work, except that justifying the RPA, the transverse fermion wave vector merely provides a convenient way of counting the chains. It should be noted that a system with interactions $\overrightarrow{\mathrm{S}}_{i \alpha} \cdot \overrightarrow{\mathrm{S}}_{j \beta}$ between chains $\alpha$ and $\beta$ cannot be mapped in this way onto a corresponding fermion problem.

\section{PHONON SOFTENING AND THE MEAN-FIELD TREATMENT}

The diffuse $x$-ray scattering measurements of Moncton et al. on TTFCuBDT show a pronounced peak structure at the $\left(\frac{1}{2}, 0, \frac{1}{2}\right)$ corner of the primitive unit cell-that wave vector at which a Bragg peak appears below $T_{c}$. This peak persists up to 225 ${ }^{\circ} \mathrm{K}$, where a second structural transition occurs. We interpret the peak as due to diffuse scattering of a relatively soft phonon at this wave vector. At first sight it would be tempting to identify this softening with a pretransitional effect. However the softening persists up to temperatures greater than the characteristic magnetic interaction temperature $\left(J=77^{\circ} \mathrm{K}\right)$. Furthermore it is three dimensional (i.e. softening about a point in reciprocal space, rather than on a plane as characteristic of one-dimensional softening), whereas the magnetic interaction is thought to be one-dimensional-an interpretation strongly borne out by the excellent agreement of the susceptibility with the Bonner-Fisher ${ }^{9}$ one-dimensional calculations. We are hence forced to conclude that the effect is not solely due to the spin interactions. We will therefore take the bare phonon frequency to include this softening - assumed essentially temperature independent - and calculate the additional effects at low temperatures due to the spin interaction. Unfortunately the bare phonon spectrum $\Omega_{0}(\vec{q})$ is not given in detail by these measurements. We will take for the spectrum near the point $\vec{q}_{0}=\left(\frac{1}{2}, 0, \frac{1}{2}\right)$,

$$
\Omega_{0}^{2}(\overrightarrow{\mathrm{q}})=\Omega_{0}^{2}\left[1+\xi_{\mathrm{ph}}^{2}\left(\overrightarrow{\mathrm{q}}-\overrightarrow{\mathrm{q}}_{0}\right)^{2}\right]
$$


The simplest interpretation of the experiments is that the scattering is mainly from a single-phonon branch. Then the assumption that for temperatures large compared with the phonon frequency a scattering intensity proportional to $\Omega_{0}^{2}(\overrightarrow{\mathrm{q}})$ (as expected for diffuse scattering), suggests that $\Omega_{0}^{2}$ is at least a factor of 3 smaller than given by a typical phonon frequency. Note that contributions to the background from other modes will increase the estimate of this factor. From the width of the scattering peaks we estimate $\xi_{\mathrm{ph}} \sim 10 \AA$. We use this pre-existing soft phonon to justify the mean-field random-phase approximation except for a critical region near $T_{c}$, which we estimate to be small using the Ginzburg criterion. ${ }^{14}$

The phonon frequency in the presence of the spin interactions is given by the pole of the exact phonon Green's function $\mathrm{D}(q, \Omega)$ given by Dyson's equation [Fig. 1(a)]

$$
D=D_{0}+D_{0} \Pi D \text {, }
$$

where $D_{0}$ is the noninteracting Green's function

$$
D_{0}(q, \Omega)=\left[\Omega^{2}-\Omega_{0}^{2}(q)+i \eta\right]^{-1}
$$

and $\Pi$ is the exact polarizability of the spin (pseudofermion) system. The random-phase approximation corresponds to taking the polarizability to be that calculated in the absence of fermionphonon coupling, i.e., in diagrammatic language it is the "bubble" containing no phonon lines [Fig. 1(b)] (for simplicity we neglect the four fermion interactions in this section). For a one-dimensional fermion system there is no general "Migdal's theorem" to suggest RPA should be valid: the diagrams neglected in RPA are also logarithmically divergent in onedimension. ${ }^{17}$ However, as pointed out by Chui et al. ${ }^{18}$ in a slightly different context, the diagrams not contained in the RPA expansion [the simplest example is probably Fig. 1(c)] involve a summation over the coordinates of the internal phonon Green's function. In our case, as may be seen by evaluating Fig. 1(c), this introduces small factors of order

$$
\left\langle\frac{g^{2}\left(\overrightarrow{\mathrm{q}}_{0}+\overrightarrow{\mathrm{q}}_{\perp}\right)}{\Omega_{0}^{2}\left(\overrightarrow{\mathrm{q}}_{0}+\overrightarrow{\mathrm{q}}_{\perp}\right)}\right\rangle_{\text {av }} / \frac{g^{2}\left(\overrightarrow{\mathrm{q}}_{0}\right)}{\Omega_{0}^{2}\left(\overrightarrow{\mathrm{q}}_{0}\right)}
$$

together with logarithmic corrections, relative to the RPA diagram of the same order [Fig. 1(d) for the example 1c] which does not involve a summation over the internal phonon. In Eq. (3.4) \langle\rangle$_{\mathrm{av}}$ indicates an average over wave vectors $\overrightarrow{\mathrm{q}}_{\perp}$ transverse to the chain direction, and $\vec{q}_{0}$ is the soft-phonon wave vector. The magnitude of expression (3.4) depends on the amount of softening of the bare phonon, not yet known in detail. It should be, at worst, 0.3 corresponding to the minimum softening estimated above. Thus although the diagrams not kept in RPA are also logarithmically divergent, they are reduced in magnitude by the important momentum dependence of the effective interaction due to the phonons. The usual argument for truly one-dimensional systems with momentum-independent interactions, that the diagrams left out in RPA are in fact of the same magnitude as the RPA diagrams and just such as to suppress the transition temperature to zero temperature, is not true here. In this case we may use RPA to discuss qualitatively the transition, and if the parameter (3.4) is sufficiently small to give a good quantitative description, as well.

Near enough to the transition temperature predicted by RPA, the "pile up" of contributions from the small denominators of the phonon Green's functions in "fluctuation" diagrams ${ }^{17}$ will however invalidate the RPA. Within this critical region the mean-field treatment will not be good. A quantitative estimate of the mean-field character of the transition is given by the Ginzburg parameter ${ }^{14}$

$$
\epsilon_{c}=\frac{1}{32 \pi^{2}}\left(\frac{1}{\Delta C \xi_{0}^{3}}\right)^{2}
$$

in three dimensions, which is an estimate of the fractional width near $T_{c}$ where such fluctuations are important. In this expression $\Delta C$ is the specific-heat jump per volume at $T_{c}$, and $\xi_{0}$ is the coherence length. Here we take $\Delta C$ from experiment ${ }^{18}$ : $\Delta C=1.0 \mathrm{~N}\left(T_{c} / J\right)$ with $N$ the number of formula weights per volume, and leave comparison with theory to later. For $\xi_{0}$ we use the inverse width of the pre-existing soft phonon $\xi_{\text {ph }}$ (this neglects any additional narrowing parallel to the chain direction near $T_{c}$ which makes $\epsilon_{c}$ even smaller). This yields

$$
\epsilon_{c}=2\left[\frac{1}{8} \pi^{2}\left(J / T_{c}\right) f\right]^{2},
$$

where $f$ is the fraction of the reciprocal unit cell (volume $V_{R}$ ) occupied by the pre-existing soft phonon [to half height in $\Omega_{0}^{-2}(\overrightarrow{\mathrm{q}})$ ], that is $\left(2 / \xi_{\mathrm{ph}}\right)^{3} / V_{R}$. From the estimate of $\xi_{\mathrm{ph}}$ we find $\epsilon_{c} \sim 0.04$. Thus except for a rather small region $\sim 0.04 T_{c}$ near $T_{c}$ a mean-field treatment should be good, and in particular we expect the RPA to be good. Note the small value of $\epsilon_{c}$ comes from the small parameter $f \sim 0.02$ : in the absence of a pre-existing, threedimensionally soft phonon $\epsilon_{c}$ would be of order 1 . The Ginzburg criterion gives only a rough estimate for the critical region; nevertheless experiments ${ }^{4,18}$ also seem to indicate a critical width of this order.

\section{CALCULATION OF POLARIZABILITY}

With the encouragement of the theoretical justification for the RPA of Sec. III, we now attempt to calculate the polarizability $\Pi$-the linear response of the spin system to the phonon coupling-more accurately than was previously thought useful. From the form of the pseudofermion Hamiltonian and the 
work of Solyom ${ }^{19}$ and Luther and Peschel ${ }^{20}$ it is clear that the four-fermion interactions will lead to a polarizability more divergent as $T \rightarrow 0$ than the $\ln J / T$ of the Hartree approximation. A quantitative treatment is made difficult since the interactions $\sim J_{z}$ are of the same size (in the Heisenberg limit) as the bandwidth $\sim J_{1}$, and the range of the potential is comparable to the inverse Fermi wave vector: there are no obvious small parameters on which to base a perturbation theory.

Our method of calculation of the phonon-induced transition is based on the work of Luther and Peschel on the uniform Heisenberg chain. ${ }^{8}$ Their method is to approximate the pseudofermion Hamiltonian by the exactly soluble Luttinger-Tomanaga model. The basic idea is that responses that are divergent due to anomalous infrared properties of the Fermi sea (note in the Hartree approximation $\Pi$ is divergent due to a constant density of states for zero-energy $2 k_{F}$ excitations across the Fermi sea) can be approximately calculated by modelling the Hamiltonian with a soluble form that maintains the essential features (Fermi velocity, interaction potential matrix elements) in the region near the Fermi surface leading to the infrared divergence. Within the model the four-fermion interactions can be exactly included, and no perturbation expansion is needed. In addition the response to the phonon coupling can, even in practice, be very easily calculated. On the other hand the approximations used are in general rather uncontrolled. For small values of $J_{z} / J_{1}$ we would expect the physics to be dominated by excitations near the Fermi surface, and an approximation preserving the properties near the Fermi surface should be good. But for $J_{z} \sim J_{\perp}$ excitations well away from the Fermi surface are also involved, and the validity of the approximation is not so clear. In the particular case of the Heisenberg limit, however, it is possible to test the accuracy by comparison with some exactly known results. Alternatively one may choose the parameters of the model Hamiltonian to reproduce these results, and this, it will be seen, allows an exact calculation of some quantities of interest, providing certain "universality" assumptions are valid. Other results will depend on details of the Hamiltonian, such as band structure and wave vector dependence of the potential, that we have been forced to approximate: such results will be only approximate. In particular numerical prefactors are found to depend on a high momentum cutoff needed to give finite answers.

It will be clear from the above discussion that we approach the Heisenberg limit from an $X Y-Z$ model; that is, it is assumed that the limit $J_{z} / J_{1} \rightarrow 1$ from below may be taken and that it correctly gives the Heisenberg behavior. This procedure is somewhat dangerous, since for any $J_{z} / J_{1}$ greater than one there is a gap in the excitation spectrum, and at sufficiently low temperatures the behavior is Ising-like. However exact results ${ }^{21}$ show that the long-wavelength excitation spectrum in the Heisenberg case is linear, as in the $X Y$ limit $^{22}\left(J_{z} / J_{1}=0\right)$. Therefore we follow previous approaches in treating the Heisenberg chain as a continuation from the $X Y$ chain, but calculate the effect of the $Z$ interactions in a better approximation than previous work.

We will not go through the derivation of the Luttinger-model approximation to the Heisenberg chain in detail here: some more details are in Appendix $\mathrm{A}$, and the procedure has been described before in the literature. ${ }^{8} \mathrm{~A}$ brief list of the approximations may be useful however. The (exact) pseudofermion Hamiltonian is Eq. (2.6). The cosine energy band of $H_{0}$ is first replaced by linear bands maintaining the correct velocity $v_{F}$ at the Fermi surface. It is convenient for computational purposes to consider two sets of fermions ( 1 and 2 ) each with a linear band extending to $\pm \infty$ with velocities $v_{F}$, and $-v_{F}$, respectively. All the levels below the Fermi level are filled in the noninteracting ground state and the Fer$\mathrm{mi}$ wave vector is fixed by the total spin:

$$
S^{z}=\left(s k_{F} / \pi-\frac{1}{2}\right) N .
$$

A similar approximation is to replace the interaction $V(q)$ by $V(0)=J$ for scattering near the Fermi surface $(q \approx 0)$ and by $V(\pi / s)=-J$ for scattering across the Fermi surface $\left(q \approx 2 k_{F}=\pi / s\right)$. This latter term corresponds to backward scattering but it can be rewritten ${ }^{23}$ as forward scattering for these spinless fermions [Fig. 2(a)]. In addition fermion umklapp scattering processes [Fig. 2(b)] are canceled by the exchange interaction [Fig. 2(c)], and are not important. ${ }^{24}$ This helps justify the replacement of the periodic band structure by linear bands.

These approximations lead to a Hamiltonian essentially that of the Luttinger model, which has been shown to be exactly soluble by Mattis and Lieb. ${ }^{7}$ The detailed form is written in the Appendix. We note here merely a slight difference between our expression and that of Luther and Peschel, ${ }^{8}$ due to their neglect of scattering events with momentum transfer of order zero. This leads to differences in some of the calculations, but the final results for the Heisenberg limit are not altered.

The fermion-phonon coupling is of the form ${ }^{25}$

$$
\begin{aligned}
H_{\mathrm{ph}}= & \frac{1}{\sqrt{N}} \sum_{q} g(q)\left(1-e^{i q s}\right) Q(q) \\
& \times\left(\sum_{l} e^{i q l s} \vec{S}_{l} \cdot \vec{S}_{l+1}\right) .
\end{aligned}
$$

The relevant polarizability is therefore

$$
\begin{aligned}
\Pi(q, \omega)= & \left|g(q)\left(1-e^{i q s}\right)\right|^{2} \int d t e^{i \omega t} \sum_{l} e^{-i q l s} \\
& \times\left\{-i \Theta(t)\left\langle\left[\left(\overrightarrow{\mathrm{S}}_{l} \cdot \overrightarrow{\mathrm{S}}_{l+1}\right)_{t},\left(\overrightarrow{\mathrm{S}}_{0} \cdot \overrightarrow{\mathrm{S}}_{1}\right)_{t=0}\right]\right\rangle\right\} .
\end{aligned}
$$



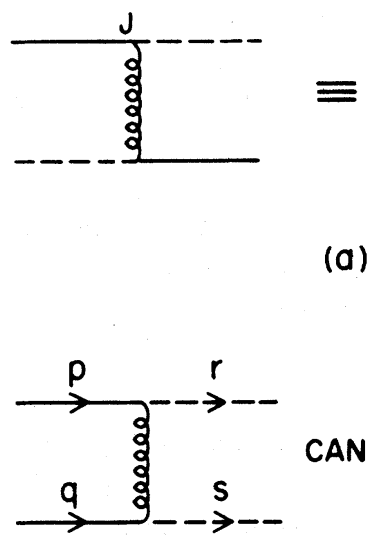

(a)

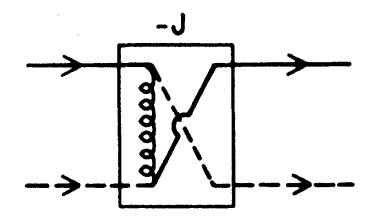

(b)

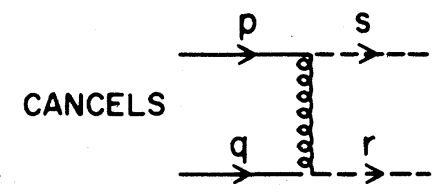

(c)

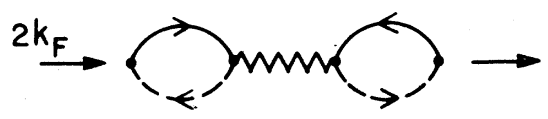

(d)

FIG. 2. Approximations in deriving the Luttinger-

Tomanaga representation (see text). The curly line denotes the four-fermion interaction; (d) should be compared with the "exchange" diagram in Fig. 1(c).

It is here we must be rather careful to include the phonon-fermion umklapp processes. Note first that replacing the sum in Eq. (4.3) by an integral

$$
\sum_{l} e^{-i q l s} \rightarrow \int \frac{d x}{s} e^{-i q x}, \text { with } x=l s
$$

gives the polarizability $\Pi_{c}(q, \omega)$ we would calculate for a continuum, linear band model. For the original periodic band structure the correct polarizability to include phonon umklapp processes is

$$
\begin{aligned}
\Pi\left(2 k_{F}+\delta q, \omega\right)= & \Pi_{c}\left(2 k_{F}+\delta q, \omega\right) \\
& +\Pi_{c}\left(2 k_{F}+\delta G-\delta q,-\omega\right),
\end{aligned}
$$

where $\delta G=2 \pi / s-4 k_{F}$ in general and is zero in the half-filled case. The two processes contributing in the noninteracting limit are shown in Fig. 3, and the generalization is obvious. For the important $2 k_{F}$, zero-frequency polarizability for the half-filled band Eq. (4.4) leads to an additional factor of 2 in the divergent response compared with a band well away from half full. The consequent strong enhancement of the transition temperature for the half-filled case is well known.

The transformation to the pseudofermion representation of adjacent spin operators in Eq. (4.2) is partic-
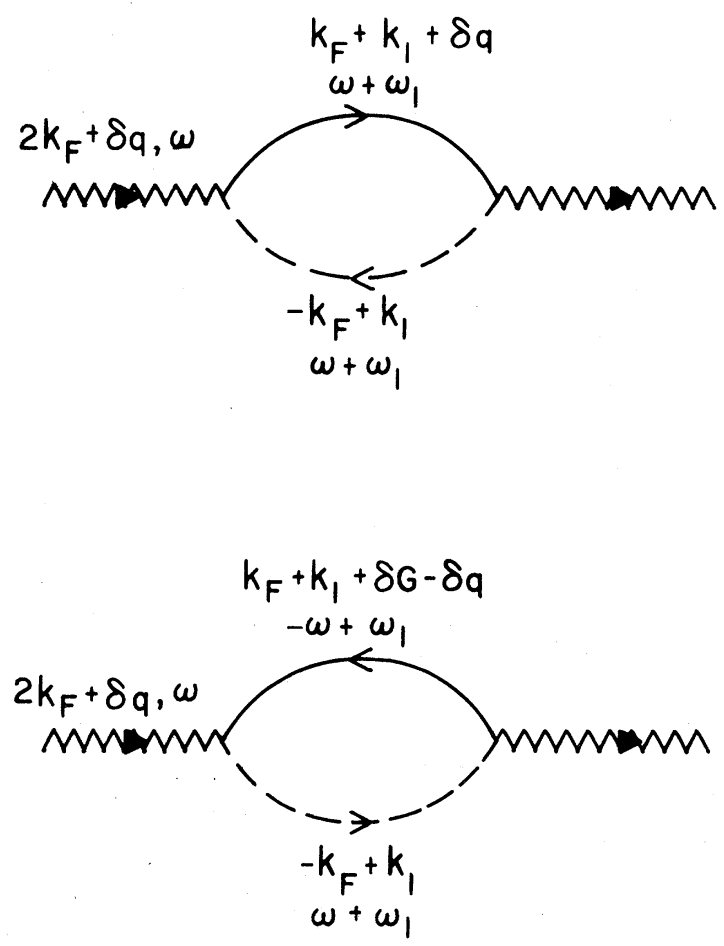

FIG. 3. (a) Normal and (b) Umklapp processes contributing to $\Pi$ for noninteracting fermions. Here $\delta G=2 \pi s^{-1}-4 k_{F}$ is zero for a half-filled band.

ularly easy, and it is then rather straightforward to evaluate the response function required using the procedure of Luther and Peschel: ${ }^{8}$ : we do this in Appendix A. For convenience we define a retarded "energy response function" by

$\chi_{\epsilon}(x=l s, t)=-i \Theta(t)\left\langle\left[\left(\vec{S}_{l} \cdot \vec{S}_{l+1}\right)_{t},\left(\vec{S}_{0} \cdot \vec{S}_{1}\right)_{t=0}\right]\right\rangle$

We calculate this to be

$$
\begin{aligned}
\chi_{\epsilon}(x, t)= & -\Theta(t) 9 s^{2} \frac{\cos \left(2 k_{F} x\right)}{(2 \pi \alpha)^{2}} \\
& \times[Q(c t-x) Q(c t+x)]^{-1 / 2 \theta} \times\left(1+\frac{s^{2}}{\alpha^{2}}\right)^{1 / 4 \theta-\theta} \\
& \times \sin \left(\frac{\pi}{4 \theta}[\operatorname{sgn}(c t-x)+\operatorname{sgn}(c t+x)]\right)
\end{aligned}
$$

where $Q(y) \approx(c / \pi \alpha T) \sinh (\pi T y / c), \alpha$ is a cutoff parameter of order $s / \pi, c$ is the renormalized Fermi velocity, and $\theta$ is a characteristic exponent, and we have dropped terms leading to higher-order contributions to the $2 k_{F}$ Fourier transform. In deriving this result we have used the rotational invariance of the Heisenberg Hamiltonian to relate the parts of $\chi_{\epsilon}$ involving $S^{z}$ to the more easily calculated transverse components. The resulting Eq. (4.6) is only strictly 
correct for $|x \pm c t| \gg \alpha$.

The exponent $\theta$ in the Heisenberg limit is calculated by the methods of Luther and Peschel to be 0.85 (see Appendix A). However it is believed ${ }^{8}$ that the correct answer here is $\theta=1$. This result is necessary for the equality of the calculated ${ }^{8}$ long distance behavior of the simple correlation functions $\left\langle S^{y}(x) S^{y}(0)\right\rangle$ and $\left\langle S^{z}(x) S^{z}(0)\right\rangle$ required by the rotational symmetry of the exact Heisenberg Hamiltonian, and is also suggested by the relationship of $X Y Z$ chains to the Baxter model. ${ }^{8,26}$ The important assumption is that although $\theta$ is not given accurately by the Luttinger-model approximation, the relationship between the exponents of various divergent correlation functions (scaling relations), given by the model in terms of $\theta$, are also true for the exact Hamiltonian. After fixing $\theta$ by known properties of simple correlation functions, we can evaluate the exponents for the more complicated ones of interest exactly.

Similarly we estimate $c=1.15 \mathrm{Js}$, smaller by about $25 \%$ than the value of the long-wavelength spin-wave speed $\frac{1}{2} \pi J s$ given by the exact calculations of des Cloiseaux and Pearson. ${ }^{21}$

The procedure we adopt, to best make use of the known results, is to use the calculated answers to give the form of the response function in terms of $\theta$ and $c$, and then to substitute the exact values $\theta=1$, $c=\frac{1}{2} \pi J s$. We may be quite confident that this gives the correct exponents for the divergent polarizability (our most important results) and hope also to get some useful information on its functional form (at least at large distances) and numerical prefactors, although the latter will probably not be accurate. The hope is clearly that the Luttinger type Hamiltonian does give a good representation for the divergent small $\omega, q, T$ behavior, but that the $v_{F}$ and potential matrix element may be changed from the naive values used by renormalizing effects from scattering processes well away from the Fermi surface. As far as we know this has not been justified.

We then find for the Heisenberg chain $(\theta=1)$ for $q$ near $2 k_{F}$,

$$
\begin{aligned}
\Pi_{c}(q, \omega) & =-0.37\left|\left(1-e^{i q s}\right) g(q)\right|^{2} \\
& \times I_{1}\left(\frac{\omega+c\left(q-2 k_{F}\right)}{2 \pi T}\right) \\
& \times I_{1}\left(\frac{\omega-c\left(q-2 k_{F}\right)}{2 \pi T}\right) \frac{1}{T},
\end{aligned}
$$

where

$$
\begin{aligned}
I_{1}(k) & =\frac{1}{2 \pi} \int_{0}^{\infty} e^{i k x}(\sinh x)^{-1 / 2} d x \\
& =\frac{1}{\sqrt{8 \pi}} \frac{\Gamma\left(\frac{1}{4}+\frac{1}{2} i k\right)}{\Gamma\left(\frac{3}{4}+\frac{1}{2} i k\right)}
\end{aligned}
$$

and $I_{1}(0)=0.590$. In particular for the half-filled band, and taking $q \rightarrow 2 k_{F}, \omega \rightarrow 0$ :

$$
\Pi\left(2 k_{F}, 0\right)=0.26 \times 4\left|g\left(2 k_{F}\right)\right|^{2} T^{-1} .
$$

This result is independent of the renormalization of the Fermi velocity $c / v_{F}$ and is only weakly dependent on the choice of cutoff (the prefactor varies by less than $20 \%$ for cutoffs over the range $0.3<\alpha / s<1.4$ : for the sake of definiteness we make the natural choice $\alpha=s / \pi$ ).

Hence we find that for the Heisenberg case the $2 k_{F}$ polarizability diverges as $T^{-1}$ rather than the $\ln J / T$ predicted by the Hartree approximation. The theory predicts for the inbetween (easy plane) case $0<J_{z}<J_{1}$

$$
\Pi\left(2 k_{F}, 0\right) \sim(1 / J)(J / T)^{2-1 / \theta}
$$

with $\theta$ given by eq. (A17b):

$$
\theta=\frac{1}{2}+(1 / \pi) \sin ^{-1}\left(J_{z} / J_{1}\right)
$$

For the $X Y$ limit $\left(\theta=\frac{1}{2}\right)$ the logarithmic dependence is exact.

\section{CONSEQUENCES}

In this section we show the consequences of our better calculation of the polarizability. We will emphasize the qualitative changes from the results of earlier work - that is those that depend on parameters of the system such as $J, T_{c}$ etc. As we have seen numerical prefactors will probably not be accurately given by our calculations. It turns out that many results are changed by factors involving $T_{c} / J$. In a truly weak coupling system $\left(T_{c} / J \ll 1\right)$ this would give very large effects; in TTFCuBDT the ratio is only one sixth and hence only relatively small, but probably measurable, differences arise. It is interesting, however, that many thermodynamic quantities are given (to within factors of order unity) by essentially the same expressions as in the Hartree approach, even though the underlying microscopic expansion is quite different.

\section{A. $T_{c}$ and above}

As the temperature is reduced the phonons become progressively softened until at $T_{c}$ a phonon frequency becomes zero. This will be for the $2 k_{F}=\pi / s$ phonon:

$$
0=\Omega^{2}\left(2 k_{F}\right)=\Omega_{0}^{2}\left(2 k_{F}\right)+\Pi\left(2 k_{F}, 0, T_{c}\right)
$$

The enhanced divergence of the polarizability we find, gives a larger transition temperature for a given 
coupling constant, and a quite different linear dependence on the coupling constant. In terms of the dimensionless coupling constant $\lambda=4 g^{2} / \pi J \Omega_{0}^{2}$ with $g=g(\pi / s)$ result $(4.9)$ gives

$$
T_{c} / J=0.8 \lambda \text {. }
$$

This should be compared with the result given by the Hartree approach $T_{c} / J=1.4 \exp (-0.6 / \lambda)(\operatorname{Ref} .27)$.

In practice $\lambda$ is estimated from the measured $T_{c}$ : thus for TTFCUBDT we find a smaller coupling constant $(\lambda=0.19)$ than the Hartree approach

$(\lambda=0.29)$. Because of possible errors in the numerical factors the ratio $\left(J / T_{c}\right) / \ln \left(1.4 J / T_{c}\right) \sim 3$ may give a better guide to the magnitude of the change. We remark in passing that the apparent absence of phonon corrections to the Bonner-Fisher ${ }^{9}$ susceptibility may be understood from the small average spinphonon coupling constant, which again is $\lambda$ reduced by roughly $\Omega_{0}^{2}\left(2 k_{F}\right) /\left\langle\Omega_{0}^{2}\right\rangle_{\text {av }}$ due to summation over the Brilliouin zone. For example a high-temperature expansion gives for the susceptibility

$$
\begin{aligned}
\chi T & =\frac{1}{4}-\frac{1}{8} \frac{J}{T}\left(1+\frac{1}{2 J} \times \sum_{q} \frac{|g(q)|^{2}}{\Omega_{q}^{2}}(1-\cos q)^{2}\right) \\
& \approx \frac{1}{4}-\frac{1}{8} \frac{j}{T}\left(1+\frac{3 \pi}{16} \bar{\lambda}\right),
\end{aligned}
$$

where $\bar{\lambda}$ is a weighted average of the coupling constant over the zone.

Also arising from the enhanced divergence of the polarizability we find an enhanced rate of softening of the $2 k_{F}$ phonon. Thus near $T_{c}$,

$$
\Omega^{2}=\Omega_{0}^{2}\left(T / T_{c}-1\right)
$$

(cf. the Hartree result $\Omega^{2}=\Omega_{0}^{2}\left(T / T_{c}-l\right) / \ln \left(1.4 J / T_{c}\right)$. The slope is increased by a factor $\ln \left(1.4 J / T_{c}\right)$ which although only about 2 in TTFCuBDT, should provide a test of our theory if more accurate measurements of the phonon spectrum are made. There are similar effects on the narrowing of the phonon dip near $T_{c}$, but comparison with experiment ${ }^{4}$ is complicated by possible additional narrowing along the chain direction due to the wave vector dependence of the polarizability.

\section{B. Below but close to $T_{c}$}

To describe the thermodynamic properties in this region we take advantage of the mean-field behavior and use a Ginzburg-Landau expansion for the free energy:

$$
\begin{aligned}
F=\alpha & -\left[1-T / T_{c}-\frac{1}{2} \eta\left(h / T_{c}\right)^{2}\right]\left(\Delta / T_{c}\right)^{2} \\
+ & \left.\frac{1}{2} b\left(\Delta / T_{c}\right)^{4}\right\}
\end{aligned}
$$

where $\Delta$ is the "order parameter" proportional to the lattice distortion in the Ginzburg-Landau region, $h=\mu H$ gives the coupling to a magnetic field to lowest order in $\Delta^{2}, \alpha$ is an energy, and $\eta$ and $b$ are numbers to be calculated. The specific-heat jump, decrease in susceptibility, depression of $T_{c}$ by a magnetic field and growth of the order parameter are then all given, using obvious manipulations, to leading order in $\left(1-T / T_{c}\right)$ by $\alpha, b$ and $\eta$.

For the Hartree calculation $\Delta$ may be chosen as the gap in the pseudofermion spectrum $\Delta=p \Delta_{0}$ with $\Delta_{0}$ the "bare gap" $\Delta_{0}=2 g Q / \sqrt{N}$ (for the half-filled band case). The expansion Eq. (5.5) is then BCS-like with

$$
\alpha=\frac{1}{2} N(0) T_{c}^{2}=N T_{c}^{2} / 2 \pi p J
$$

and

$$
b=7 \zeta(3) / 8 \pi^{2} .
$$

In our calculation the second-order terms are already known, since to this order

$$
F=\frac{1}{2} Q^{2}\left[\Omega_{0}^{2}+\Pi(T)\right] .
$$

Expanding $\Pi$ about $T_{c}$ then gives

$$
F=0.8 \frac{N T_{c}^{2}}{2 \pi J}\left[-\left(1-\frac{T}{T_{c}}\right)\left(\frac{\Delta_{0}}{T_{c}}\right)^{2} \frac{J}{T_{c}}\right],
$$

where $\Delta_{0}$ is again the same "bare gap" proportional to $Q$. Note the additional factor of $J / T_{c}$ due to the enhanced divergence of $\Pi$. The further terms in the expansion may be calculated in principle from more complicated correlation functions: the first term (in $\Delta_{0}^{2}$ ) is given by $\mathrm{II}$; the second (in $\Delta_{0}^{4}$ ) involves a nonlinear response to the lattice distortion given by a four-point correlation function. An analysis of this, and higher terms (Appendix B) shows the expansion parameter to be $\Delta=\sqrt{J / T} \Delta_{0}$ : the series is then as Eq. (5.5) with $b$ (and successive coefficients) pure numbers. This then gives

$$
\alpha=0.8 N T_{c}^{2} / 2 \pi J
$$

close to ( $-30 \%$ larger than) the Hartree result. The value of coefficient $b$ is hard to evaluate, and in any case will be cutoff dependent in our theory: we have not attempted to calculate it. However we may conclude that the form of the Ginzburg-Landau expansion, and hence the thermodynamic quantities including $\Delta$ mentioned above are essentially the same as in the Hartree approach (to within factors of order unity we have not evaluated): however the relationship of $\Delta$ to the lattice distortion is changed and at a given $T / T_{c}$ and for a given coupling constant $g$ the magnitude of the lattice distortion is of order $\sqrt{T_{c} / J}$ smaller than predicted by the Hartree theory.

The coupling to the magnetic field to leading order 
in $\Delta^{2}$ may also easily be calculated by approaching $T_{c}$ from above. The effect of a magnetic field (chosen in the $z$ direction) is to change the total $z$ spin and hence the pseudofermion filling. Thus $2 k_{F}$ is no longer equal to the wave vector of the lattice distortion which, at least in not too large fields, will remain at $\pi / s$. Thus the change in the polarizability $\Pi$ from which $\eta$ is calculated is essentially a "gradient" term. To leading order in $H^{2}$,

$$
\begin{aligned}
\Pi\left(\frac{\pi}{a}, H\right)= & \Pi\left(\frac{\pi}{a}, H=0\right) \\
& +\frac{1}{2} \frac{\partial^{2} \Pi}{\partial q^{2}}\left[\delta\left(2 k_{F}\right)\right]^{2},
\end{aligned}
$$

where $\delta\left(2 k_{F}\right)=(2 / \pi s) \mu H / J$. In this last expression we have used the zero-temperature susceptibility in the undistorted state $\chi_{N}=\left(1 / \pi^{2}\right) N \mu^{2} / J$. Introducing an "intrinsic correlation length" in the usual way,

$$
\xi_{\text {in }}^{2}=\frac{1}{2} \frac{\partial^{2} \Pi}{\partial q^{2}} / T \frac{\partial \Pi}{\partial T}
$$

and using eq. (5.7) for the energy or directly calculating the depression in $T_{c}$ gives for $\eta$ the very simple result

$$
\eta=2\left[(2 / \pi) T_{c} / J\right]^{2}\left(\xi_{\text {in }}^{2} / s^{2}\right)
$$

Computing $\xi_{\text {in }}^{2}$ from the expression for $\Pi$, Eq. (4.7) yields

$$
\left(\xi_{\text {in }}\right)^{2}=\frac{1}{2} \times 1.8(c / \pi T)^{2},
$$

where the numerical prefactor comes from ratios of the integrals $I_{1}(0), I_{1}^{\prime}(0)$, and $I_{1}^{\prime \prime}(0)$. Note that any numerical prefactors in $\Pi$ and dependence on the cutoff cancel in the ratio giving $\xi_{\text {in }}^{2}$ : the result depends only on the form of the correlation functions. We may then hope that this result is quantitatively accurate. These calculations give $\eta=0.18$ where we have used the exact spin wave velocity $c=\frac{1}{2} \pi J_{s}$. The Hartree result gives the usual onedimensional BCS form for $\xi_{\text {in }}$ :

$$
\left(\xi_{\text {in }} / s\right)^{2}=\frac{7}{16} \zeta(3)\left(p J / \pi T_{c}\right)^{2}
$$

leading to $\eta=0.12$. Comparison with experiment is made difficult by an apparent inconsistency of the experiments with relationships predicted by the Ginzburg-Landau expansion. Thus calculating $\eta$ from the measured susceptibility decrease below $T_{c},{ }^{1}$ and the measured specific-heat jump ${ }^{18}$ leads to $\eta=0.25$ (Ref. 28); on the other hand from the direct measurements of the depression of $T_{c},{ }^{29}$ apparently well fit by an $H^{2}$ dependence, we would estimate $\eta=0.43$. These differences may simply indicate the inaccuracy of these rather difficult experiments. Note however our result is $50 \%$ larger than the Hartree result, in better agreement with experiment.

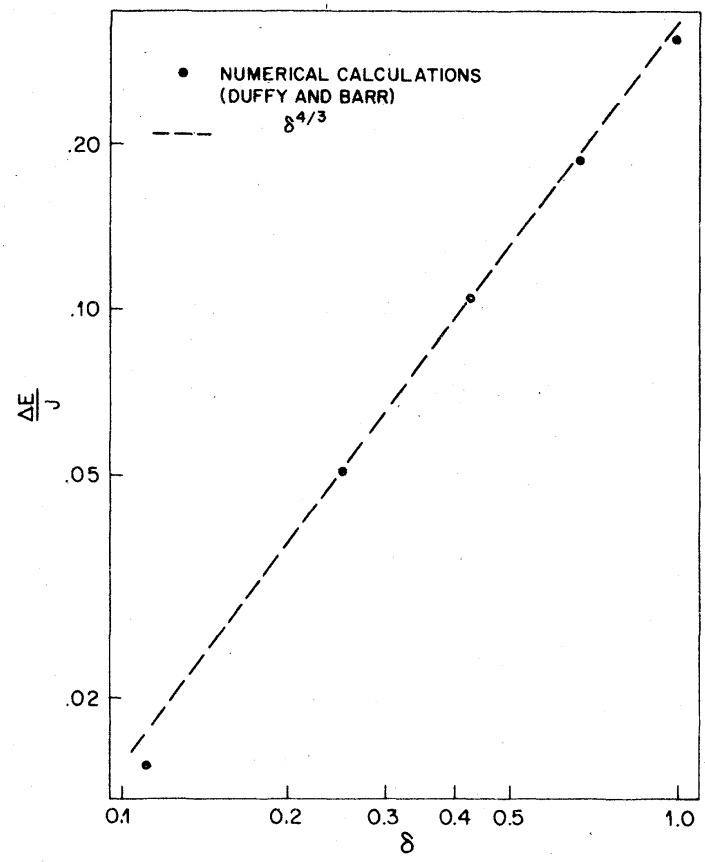

FIG. 4. Decrease in energy of spin system for a lattice distortion $\delta=2 \mathrm{gQ} / \sqrt{N} J$. The points are from the numerical calculations of Duffy and Barr (Ref. 35) using $\delta=(1-a) /(1+a)$ and $\Delta E / J=\left|\epsilon_{0} /(1+a)-\frac{1}{2} \epsilon_{0}\right|_{a=1}$, where $\epsilon_{0}$ and $a$ are the parameters of Ref. 33 .

\section{The "incommensurate" transition}

The spin-Peierls system provides a particularly interesting example of a one-dimensional (pseudo-) fermion system in that the filling of the band can be changed simply by changing the total spin with an applied magnetic field. As the filling is changed, initially the umklapp processes still favor the distortion at wave vector $\pi / s$. This is no longer exactly at $2 k_{F}$, the singular point of the fermion response, and $T_{c}$ is reduced. This is just the depression of $T_{c}$ by a magnetic field described for small $H^{2}$ by $\eta$. At a critical filling, and a corresponding critical $T_{c}$, the transition temperature to a phase with lattice distortion at a wave vector $q \neq \pi / s$ rises above that calculated for the commensurate state. The transition is then to a phase with lattice distortion at wave vector $q$ which moves continuously away from $\pi / s$ as the filling is further changed, until eventually $q$ becomes equal to $2 k_{F}$ for the filling, with a transition temperature corresponding to a coupling constant $\lambda$ reduced to one-half the initial value: umklapp processes no longer contribute. This qualitative description follows from the work of Leung ${ }^{30}$ on the Peierls system. Bray $^{31}$ has applied these calculations to the spinPeierls problem described in the Hartree approximation. The description neglects the smaller energies that may lock the wave vector $q$ to other commen- 
surate values $2 \pi / n s$ with $n \neq 2$.

If there were no pre-existing phonon softening parallel to the chain direction (which does not destroy the validity of RPA) it is straightforward to repeat Leung's calculations, but using our polarizability relevant to the spin-Peierls system Eqs. (4.4), (4.7). The use of the polarizability Eq. (4.7) is not exact for fillings away from half-filled, but seems a reasonable approximation for $\mu H / J$ small. This procedure leads to rather similar predictions (cf. Bray ${ }^{31}$ ) for the critical magnetic field $\left(\mu H_{c} / T_{c 0}=1.4\right)$ and corresponding transition temperature $\left(T_{c} / T_{c 0}=0.77\right.$ where $T_{c 0}$ is the transition temperature in zero field) at which the transition wave vector begins to move away from $\pi / s$. For large enough fields, however, $T_{c}$ would saturate at $\frac{1}{2} T_{c 0}$, corresponding to the linear dependence on coupling constant (Bray would find $\left.T_{c} / T_{c 0} \rightarrow T_{c 0} / J\right)$.

Unfortunately, for TTFCuBDT the pre-existing softening of the phonon parallel to the chain at wave vector $\pi / \mathrm{s}$ gives an additional pinning energy at this wave vector. However from Eq. (5.12) and the value of $\eta$ (either experimental or theoretical estimate), and our estimate of $\xi_{\mathrm{ph}}^{2}$ below Eq. (3.1), it seems that $\xi_{\text {in }}^{2}$ is significantly larger than $\xi_{\mathrm{ph}}^{2}$. Under these conditions the additional pinning is not too large: for example, taking $\eta=0.25$ we estimate a critical transition temperature of about $T_{c} / T_{c 0}=0.74$, corresponding to a magnetic field $\mu \mathrm{H} / T_{c 0}=1.6$ which is about $150 \mathrm{kG}$ for TTFCuBDT. There is now no expectation that $T_{c}$ will saturate at $\frac{1}{2} T_{c 0}$. These estimates must be treated with caution, since our estimate of the depression of $T_{c}$ by a magnetic field is apparently too small by about a factor of 2 compared with the direct measurements.

\section{Zero temperature}

By developing the perturbation expansion in the lattice distortion to all orders, it is possible to derive new results for the zero-temperature dependence of the ground-state energy and excitation energy gap on the lattice distortion. These results are probably most convincingly shown by homogeneity requirements on the long-wavelength dependence of zero-temperature correlation functions, as used by Luther and Peschel ${ }^{8}$ in a different context. This is done in Appendix B. In this section we will derive the results more heuristically, but using already introduced quantities.

It is natural to identify the expansion parameter in the (infinite order) Ginzburg-Landau expansion $\Delta=\sqrt{J / T_{c}} \Delta_{0}$ as the real energy gap in the excitation spectrum near $T_{c}$. At low temperatures a similar relationship between the actual gap $\Delta$ and the "bare" gap $\Delta_{0}$ would be expected, but with the $T_{c}$, which enters near the transition as the cutoff in the infrared divergences due to the thermal occupation factor, presumably replaced by the actual gap $\Delta$, which assumes this role at low temperatures:

$$
\Delta \sim(J / \Delta)^{1 / 2} \Delta_{0} .
$$

This gives $\Delta \sim J^{1 / 3} \Delta_{0}^{2 / 3}$ : the energy gap at zero temperature scales as the lattice distortion to the power $\frac{2}{3}$.

The energy of the spin system at zero temperature is proportional to

$$
\int_{0}^{Q} d Q^{\prime} \int_{0}^{Q^{\prime}} d Q^{\prime \prime} \Pi_{Q}^{\prime \prime}
$$

where $\Pi_{Q}$ is the polarizability calculated with an already present lattice distortion $Q$. Again we assume $\Pi_{Q}$ is of the form given by replacing the infrared cutoff $T$ in Eq. (4.9) by the real gap $\Delta$, leading to

$$
E \sim N \frac{\Delta_{0}^{2}}{J} \frac{J}{\Delta} \sim N J^{-1 / 3} \Delta_{0}^{4 / 3}
$$

Thus we arrive at the new and interesting result that the leading term in the spin dimerization energy of a spin- $\frac{1}{2}$ Heisenberg chain is proportional to the lattice distortion to the power $\frac{4}{3}$. This result is rather close to the approximate bounds on this energy recently calculated by renormalization group techniques. ${ }^{32}$ It is also, perhaps rather surprisingly, in rather good agreement with numerical calculations of the energy of a dimerized Heisenberg chain done, for rather large distortions, by Duffy and Barr ${ }^{33}$ (see fig. 4).

\section{SUMMARY}

We have developed a new theory of the spinPeierls transition in Heisenberg chains, in which we follow previous work ${ }^{3}$ in using the random-phase approximation for the spin-phonon coupling, but in which we calculate the response of the spin system to a lattice distortion in a much better approximationone that, for the first time, includes the important differences between the Heisenberg and $X Y$ interactions. We use the Jordan-Wigner transformation to a pseudofermion representation as in the previous work, but take account of the large four-fermion interactions by an approximate, but nonperturbative, method that should incorporate the essential physics. We find the relevant linear response of the spin system to diverge as $T^{-1}$ at low temperatures, compared with $\ln J / T$ of the Hartree approach used before. This leads to a linear dependence of the transition temperature $T_{c}$ on the coupling constant, and an enhancement of $T_{c}$ for a given coupling constant. Other quantities, such as the gap in the excitation spectrum and the energy of the spin system for a given lattice distortion and coupling constant, are also predicted to be enhanced. These results follow from our calculation of the divergent response of the spin system to a lattice distortion $Q$, which we have done 
in detail to order $Q^{2}$ and in outline only to higher orders. Nevertheless, predictions of characteristic "signatures" of the transition, such as the specific heat jump, decrease in magnetic susceptibility and temperature dependence of the lattice distortion (normalized to zero temperature), are not significantly altered from the Hartree approach. [Also it should be remarked that the coupling constant is usually not known a priori and must be estimated from the measured transition temperature. This has the unfortunate consequence that in comparing our predictions with those of the Hartree calculation, and using the coupling constant estimated within each theory from the known $T_{c} / J$, many of the enhancement factors are typically of order $\ln J / T_{c}$ rather than some power of $J / T_{c}$ as might at first be expected, and consequently less easy to detect.] The underlying microscopic description of the transition is quite different, however. For example, the instability driving the spin-Peierls transition - the decrease in the zero temperature energy of the spin system due to a static lattice distortion $Q$-goes as $Q^{4 / 3}$ instead of the $Q^{2} \ln Q$ of the Hartree calculation of Pytte. ${ }^{3}$

The relevance of our calculation falls into two parts. First we have developed the theory of the spin-Peierls transition for a model system of chains of spin- $\frac{1}{2}$ with nearest-neighbor antiferromagnet Heisenberg interactions within the chains, immersed in a three-dimensional lattice in which planes of atoms (not parallel to the chain directions) are constrained by large lattice forces to move together. This latter feature validates the RPA mean-field treatment of the phonons.

Second we suggest our calculations should give a good description of the observed transition in TTFCuBDT. We briefly describe the important results that justify this claim (Jacobs et al. ${ }^{2}$ have covered some of these points in more detail). Although an investigation of the crystal structure suggests no obvious one dimensionality, the very good agreement between the measured magnetic susceptibility and the Bonner-Fisher ${ }^{9}$ calculations is evidence for the spin- $\frac{1}{2}$ chains interacting onedimensionally and with an isotropic interaction. The corresponding gold compound (which has a similar $J$ and crystal structure) follows ${ }^{2}$ these predictions right down to $2^{\circ} \mathrm{K}$-where again, presumably, a spinPeierls transition occurs. Since the magnetic interactions in the two compounds are probably quite similar, this puts a low limit on the spin interactions that are not one-dimensional or not isotropic (or at least are Ising-like). The linear specific heat ${ }^{18}$ at lower temperatures also confirms the one-dimensionality of the spin system. There is also the question of whether the transition is in fact driven by the spin interactions, rather than simply being a lattice-driven distortion on which the spins ride. Apart from the unlikelihood of such a phonon driven transition at these low temperatures, the best argument against this is that the specific-heat jump and entropy change at the transition are indeed found ${ }^{18}$ to be roughly as calculated for the magnetic transition.

Finally we have shown that the pre-existing soft phonon in TTFCuBDT leads us to expect a meanfield transition well described by the RPA. Other authors have used an essentially one-dimensional formalism, but have justified the absence of fluctuation corrections by the rather vague assertion that the system is "really" three-dimensional. We have shown the specific features of the phonon spectrum that make this true. The importance of the role played by the pre-existing softening of the phonon leads to the speculation that a less dramatic softening in the corresponding gold compound could account for both the lowered transition temperature (despite the similar $J$ and the apparently greater width of the critical region. Extending this idea, the absence of other good examples of a simple spin-Peierls transition could be because without the coincidence of an already soft phonon the transition temperature is depressed (by the smaller coupling constant and by the greater influence of the one dimensionality) below that of a transition driven, for example, by small three-dimensional spin coupling.

\section{ACKNOWLEDGMENTS}

The authors thank W. F. Brinkman for bringing this topic to their attention; D. E. Moncton for discussions of the experimental results, both published and unpublished; and T. M. Rice, P. A. Lee, and B. I. Halperin for many invaluable suggestions. One of us (DSF) wishes to thank Bell Laboratories for its hospitality during the time most of this research was carried out and the NSF for support via a PostGraduate Fellowship.

\section{APPENDIX A}

To evaluate the response function

$$
\begin{aligned}
\chi_{\epsilon}(x=l s, t)= & -i \Theta(t) \\
& \times\left\langle\left[\overrightarrow{\mathrm{S}}_{l}(t) \overrightarrow{\mathrm{S}}_{l+1}(t), \overrightarrow{\mathrm{S}}_{0}(t=0) \cdot \overrightarrow{\mathrm{S}}_{l}(t=0)\right]\right\rangle
\end{aligned}
$$

we first make use of the rotational invariance of the Heisenberg Hamiltonian and write

$$
\begin{aligned}
\chi_{\epsilon}(x, t)=- & i \Theta(t) \\
\times & \left\{3\left\langle\left[S_{l}^{x}(t) S_{l+1}^{x}(t), S_{0}^{x}(t=0) S_{1}^{x}(t=0)\right]\right\rangle\right. \\
& \left.+6\left\langle\left[S_{l}^{x}(t) S_{1+1}^{x}(t), S_{\gamma}^{\gamma}(t=0) S_{1}^{y}(t=0)\right]\right\rangle\right\} .
\end{aligned}
$$

If we then transform the spin operators to fermion operators by the Jordan-Wigner transformation [Eq. 
(2.5)] we are left with response functions to calculate involving four-fermion creation and annihilation operators but no "ordering" operators

$$
\exp \left(\pi \cdot \sum_{j=1}^{i-1} a_{j}^{\dagger} a_{j}\right)
$$

This Appendix will deal with evaluating such expectation values of products of fermion operators. (Note that if instead of using the rotational invariance we evaluated the correlation functions involving $S^{z}$ directly, then much more complicated expectations of products of six and eight fermions would be needed. Such a calculation would be difficult, but would provide some insight into the degree to which the approximations leading to the Luttinger Hamiltonian violate the rotational invariance of the original Hamiltonian. We have not done this, but have shown that the leading divergence of the $2 k_{F}$ polarizability is indeed calculated to have the same exponent.)

The approximations described in Sec. IV lead ${ }^{34}$ to the Hamiltonian in terms of "1" and "2" operators with

$$
\begin{aligned}
& H=H_{0}+H^{\prime} \\
& H_{0}=J_{\perp} s \sum_{k} k\left(a_{1 k}^{\dagger} a_{1 k}-a_{2 k}^{\dagger} a_{2 k}\right),
\end{aligned}
$$

and

$$
\begin{aligned}
H^{\prime}= & \frac{1}{N} J_{z} \sum_{q} \rho_{1}(q) \rho_{1}(-q) \\
& +\rho_{2}(q) \rho_{2}(-q)+4 \rho_{1}(q) \rho_{2}(-q)
\end{aligned}
$$

where $\rho_{1}$ and $\rho_{2}$ are density operators defined by

$$
\begin{aligned}
& \rho_{1}(q)=\sum_{k} a_{1 k+q}^{\dagger} a_{1 k}, \\
& \rho_{2}(q)=\sum_{k} a_{2 k+q}^{\dagger} a_{2 k},
\end{aligned}
$$

satisfying a boson algebra:

$$
\begin{aligned}
{\left[\rho_{1}(-k), \rho_{1}\left(k^{\prime}\right)\right] } & =\left[\rho_{2}\left(k^{\prime}\right), \rho_{2}(-k)\right] \\
& =\frac{k L}{2 \pi} \delta_{k k^{\prime}}, \\
{\left[\rho_{1}(k), \rho_{2}\left(k^{\prime}\right)\right] } & =0 .
\end{aligned}
$$

(For a more careful definition and proof of these properties see Mattis and Lieb. ${ }^{7}$ ) Note the difference between our expression Eq. (A4) and that of Luther and Peshel $^{8}$ [Eq. (7)], who left out contributions from scattering events with momentum transfer of order zero [c.f. also Ref. (7)].

As first noted by Mattis and $\mathrm{Lieb}^{7}$ the operator

$$
T=J_{1} s \frac{2 \pi}{L} \sum_{k>0} \rho_{1}(k) \rho_{1}(-k)+\rho_{2}(-k) \rho_{2}(k)
$$

(where $L=N s$ is the length of chain) obeys the same commutation relations with the density operators as does $H_{0}$, and we may replace $H_{0}$ by $T$ to leave a Hamiltonian quadratic in density operators. The operators $\rho_{1}(k)$ and $\rho_{2}(-k)$ [more strictly $(2 \pi / L)^{1 / 2}$ $\rho_{1}(\mathrm{k}) / \sqrt{k}$ and $\left.(2 \pi / L)^{1 / 2} \rho_{2}(-k) / \sqrt{k}\right]$ are raising operators for the noninteracting Hamiltonian $H_{0}$ (or $T$ ). The Hamiltonian may be diagonalized by the (unitary) transformation

$$
\begin{aligned}
& \rho_{1}(k) \rightarrow \rho_{1}(k) \cosh \phi,+\rho_{2}(k) \sinh \phi \\
& \rho_{2}(k) \rightarrow \rho_{2}(k) \cosh \phi,+\rho_{1}(k) \sinh \phi
\end{aligned}
$$

where $\phi$ is chosen so that the $\rho_{1} \rho_{2}$ cross terms in Eq. (A5) vanish. In our case this leads to

$$
\tanh 2 \phi=-\frac{2 J_{z}}{\pi J_{1}+J_{z}}
$$

and the Hamiltonian

$$
H_{D}=\frac{2 \pi}{L} c \sum_{k>0} \rho_{1}(k) \rho_{1}(-k)+\rho_{2}(-k) \rho_{2}(k)
$$

with $c$ the renormalized Fermi velocity

$$
c=J_{\perp} s\left[\left(1+\frac{J_{z}}{\pi J_{\perp}}\right)^{2}-\left(\frac{2}{\pi} \frac{J_{z}}{J_{\perp}}\right)^{2}\right]^{1 / 2}
$$

(where we have dropped an infinite constant in $H_{D}$ ). Note again the discrepancies with Ref. 8.

In order to calculate correlation functions we must replace the lattice-site fermion operators involved in Eq. (A1) by their continuum counterpart, consistent with the infinite linear bands, defined by

$$
\psi_{1,2}(x)=(L)^{-1 / 2} \sum_{k} a_{1,2 k} e^{i k x}
$$

The correct procedure to preserve the important matrix elements is, as in the derivation of the approximate Hamiltonian, to replace each $a_{l}$ by $\sqrt{s}\left[\psi_{1}(x)+\psi_{2}(x)\right]$ with $x=s l$.

The calculation of the correlation functions is fraught with divergences due to the lack of a highmomentum cutoff, both in the single-particle spectrum and in the potential. For the results of interest to us - the leading divergence (for example in $1 / T$ ) of the polarizability - it is possible to introduce the cutoff in a rather controlled way as a highmomentum cutoff in the interaction potential in $\mathrm{H}^{\prime}$ (a momentum-dependent coefficient in $\mathrm{H}^{\prime}$ still leads to an exactly soluble problem). The cutoff in the oneparticle spectrum (which introduces some formal but probably physically unimportant problems in defining the boson representation that leads to the particularly easy method of solution) is only needed to look at 
less divergent terms. Since, however, for the physical problem of interest the cutoffs must be of the same order we will use just one cutoff parameter $\alpha \sim s$. We then use the approximate operator transformation of Luther and Peschel ${ }^{22}$ and Mattis ${ }^{37}$ :

$$
\begin{aligned}
& \psi_{1}(x) \leftrightarrow \frac{1}{\sqrt{2 \pi \alpha}} e^{i k_{F} x+\phi_{1}(x)}=O_{1}(x), \\
& \psi_{2}(x) \leftrightarrow \frac{1}{\sqrt{2 \pi \alpha}} e^{-i k_{F} x+\phi_{2}(x)}=O_{2}(x),
\end{aligned}
$$

where $\phi_{1,2}(x)= \pm \frac{2 \pi}{L} \sum_{k} \rho_{1,2}(k) e^{-i k x} k^{-1} e^{-\alpha|k| / 2}$ with the lower (upper) sign going with the $\phi_{2}\left(\phi_{1}\right)$ operator. In the limit $\alpha \rightarrow 0$ the $O_{1}$ and $O_{2}$ obey the same commutation rules with the density operators as $\psi_{1}$ and $\psi_{2}$. In addition

$\lim _{\left(x-x^{\prime}\right) / \alpha \rightarrow \infty}\left\{0_{1}(x), 0_{1}\left(x^{\prime}\right)\right\}=0$ for $x \neq x^{\prime}$

and the fermion anticommutation rules are essentially preserved for separations greater than $\alpha{ }^{36}$ Since however we will take a finite $\alpha \sim s$, the method breaks down for correlation functions involving operators of one kind (i.e., 1 or 2) at positions too close together (i.e., $<\alpha$ ). With these restrictions a general (nonvanishing) correlation function in $\psi$ operators can be written in terms of $O$ operators.

The unitary transformation Eq. (A7) that diagonalizes the density-operator Hamiltonian leads to the replacement

$$
\begin{aligned}
& \phi_{1} \rightarrow \phi_{1} \cosh \phi-\phi_{2} \sinh \phi, \\
& \phi_{2} \rightarrow \phi_{2} \cosh \phi-\phi_{1} \sinh \phi,
\end{aligned}
$$

and time evolution is then obtained by simply noting that

$$
\begin{aligned}
e^{i H} D^{t} e^{\phi_{1}(x)} e^{-i H} D^{t} & =e^{\phi_{1}(x-c t)}, \\
e^{i H D^{t}} e^{\phi_{2}(x)} e^{-i H_{D^{t}}} & =e^{\phi_{2}(x+c t)},
\end{aligned}
$$

in keeping with the interpretation of excitations travelling to the right and left, respectively. Expectation values are now trivially calculated in the diagonalized free-boson density matrix by factorizing the " 1 " and "2" parts of the correlation function and making liberal use of the identities

$$
e^{A} e^{B}=e^{A+B+[A, B] / 2}
$$

and

$$
\left\langle e^{A}\right\rangle=e^{\left\langle A^{2}\right\rangle / 2}
$$

where $A$ and $B$ are linear combinations of boson creation and annihilation operators lessentially $\rho_{1,2}( \pm$ k)].

It is found that all the correlation functions involve an exponent $\theta$ defined by

$$
2 \theta=e^{-2 \phi}=\left(\frac{1+\frac{3}{\pi} \frac{J_{z}}{J_{\perp}}}{1-\frac{1}{\pi} \frac{J_{z}}{J_{\perp}}}\right)^{1 / 2}
$$

which gives $\theta=0.85$ for the Heisenberg antiferromagnetic $J_{z} / J_{\perp}=1$. Results relating eigenvectors of the transfer matrix of the two-dimensional Baxter model $^{28}$ at $T_{c}$ to those of the quantum $X Y-Z$ chain under consideration, combined with exact results on the Baxter model suggest that in fact $^{8}$

$$
2 \theta=1+\frac{2}{\pi} \sin ^{-1} \frac{J_{z}}{J_{1}}
$$

yielding $\theta=1$ for $J_{z} / J_{1}=1$. It is interesting that with our slight modification to the approximate Hamiltonian the expansions of Eqs. (A17a) and (A17b) in small $J_{z} / J_{\perp}$ agree to second order (in contrast to the agreement only to first order found in Ref. 8). Further discussion of the difference between the value of $\theta$ evaluated from the Luttinger model and that required to satisfy exact results for the spin chain is contained in Ref. 37. Therein it is concluded that one should indeed expect a renormalization of the coupling constants on taking the continuum limit (that is on replacing the cosine bands by linear bands with a cutoff). There also the scaling relationship leading to the Eq. (A17b) is derived. As mentioned in the text the result $\theta=1$ for $J_{z} / J_{1}=1$ also follows from rotational-invariance arguments.

In addition, from Eq. (A10) we see that the continuum theory predicts a renormalized Fermi velocity

$$
c=1.15 J_{s}
$$

for the Heisenberg antiferromagnet $\left(J_{\perp}=J_{z}=J\right)$ in contrast to the exact long wavelength spin wave velocity $^{21}$

$$
c=\frac{\pi}{2} J_{s} \approx 1.57 J_{s}
$$

As stated in the text we use the form of the correlation functions calculated from the Luttinger model in terms of $c, \theta$, and $\alpha$, and use expressions (A17b) and (A18b) for the numerical values.

For reference we calculate a general equal-time correlation function in the 1 and 2 operators 


$$
\begin{aligned}
\left\langle\psi_{1}^{\dagger}\left(a_{1}\right) \psi_{1}^{\dagger}\left(a_{2}\right) \cdots \psi_{1}^{\dagger}\left(a_{n}\right) \psi_{1}\left(b_{1}\right) \cdots \psi_{1}\left(b_{n}\right) \psi_{2}^{\dagger}\left(c_{1}\right) \cdots\right. & \left.\cdots \psi_{2}^{\dagger}\left(c_{m}\right) \psi_{2}\left(d_{1}\right) \cdots \psi_{2}\left(d_{m}\right)\right\rangle \\
& =(2 \pi \alpha)^{-n-m} \exp \left[i k_{F}\left(-\sum a_{i}+\sum b_{i}+\sum c_{i}-\sum d_{i}\right)\right] \\
& \times\left(\frac{\prod_{i<j}^{n} Q\left(a_{i}-a_{j}\right) Q\left(b_{i}-b_{j}\right) \prod_{i<j}^{m} Q\left(c_{i}-c_{j}\right) Q\left(d_{i}-d_{j}\right)}{\prod_{i=1}^{n} \prod_{j=1}^{n} Q\left(a_{i}-b_{j}\right) \prod_{i=1}^{m} \prod_{j=1}^{m} Q\left(c_{i}-d_{j}\right)}\right) \\
& \times\left(\prod_{i=1}^{n} \prod_{j=1}^{m} \frac{Q\left(a_{i}-d_{j}\right) Q\left(b_{i}-c_{j}\right)}{Q\left(a_{i}-c_{j}\right) Q\left(b_{i}-d_{j}\right)}\right)^{1 / 4 \theta-\theta} \times \cdots,
\end{aligned}
$$

where the $\cdots$ is

$$
\begin{aligned}
i^{(n-m)} & \operatorname{sgn}\left(a_{1}, \ldots, a_{n}, b_{1}, \ldots, b_{n}\right) \\
& \operatorname{sgn}\left(c_{1}, \ldots, c_{m}, d_{1}, \ldots, d_{m}\right)
\end{aligned}
$$

and

$$
Q(y)=\left(1+\frac{y^{2}}{\alpha^{2}}\right)^{1 / 2} \frac{c}{\pi T y} \sinh \frac{\pi T y}{c},
$$

$\operatorname{sgn}\left(x_{1}, \ldots, x_{k}\right)$ is $(-1)^{P}$ where $\mathrm{P}$ is the number of interchanges of the $x_{i}$ 's to get to decreasing order, and none of the $a_{i}, b_{i}, c_{i}$, or $d_{i}$ are equal. The phasefactor form is valid only for all $a_{i}, b_{j}$ and $c_{i}, d_{j}$ separations large compared with $\alpha .{ }^{38}$ The correlation function at different times is only slightly more difficult to work out. The result for the correlation function given by the replacement $\psi(x) \rightarrow \psi\left(x_{i}, t_{i}\right)$ etc., is the same as Eq. (A19), with the replacement $Q\left(x_{i}\right) \rightarrow\left[Q\left(x_{i}-c t_{i}\right) Q\left(x_{i}+c t_{i}\right)\right]^{1 / 2}$, where now the phase factor depends on ordering of operators and the relative positions and times.

With these general results it is straightforward to evaluate $\chi_{\epsilon}$. It turns out that to leading order the expectation values in Eq. (A1) are identical (the difference is related to a $2 k_{F}$ pair response of the fermion system, not divergent for the sign of interaction relevant here). The dominant part of $\chi_{\epsilon}(x, t)$ [i.e., that which will lead to the dominant small- $\omega, q-2 k_{F}$ and $T$ part of $\Pi(q, \omega)$ ], is given by the above methods to be

$$
\begin{aligned}
\chi_{\epsilon}(x, t)= & -i \frac{9}{4} s^{2} \Theta(t)\left\langle\left[\psi_{1}^{\dagger}(x, t) \psi_{2}(x+s, t), \psi_{2}^{\dagger}(0,0) \psi_{1}(s, 0)\right]\right\rangle \\
= & -9 \Theta(t) \cos 2 k_{F} x\left(\frac{s}{2 \pi \alpha}\right)^{2}\left(1+\frac{s^{2}}{\alpha^{2}}\right)^{1 / 4 \theta-\theta} \\
& \times[Q(c t-x) Q(c t+x)]^{-1 / 2 \theta} \sin \left(\frac{\pi}{4 \theta}[\operatorname{sgn}(c t-x)+\operatorname{sgn}(c t+x)]\right)
\end{aligned}
$$

where we have explicitly evaluated the phase factors, and the expression is not strictly valid for $|x \pm c t| \leqslant \alpha$. Note that except for the $s$ in the arguments, the expectation value in Eq. (A21) is the same as the divergent $2 k_{F}$ density-density response function calculated by Luther and Peschel. ${ }^{20}$ The presence of the $s$ merely gives rise to the additional $\left(1+s^{2} / \alpha^{2}\right)^{1 / 4 \theta-\theta}$ factor. Since rotational-invariance arguments have been used to include the $z$ spin terms (leading to the factor 9/4) Eq. (A21) is only strictly valid for the Heisenberg chain.

The Fourier transform is easily done by substituting $u=c t-x, v=c t+x$ to give near $q=2 k_{F}$

$$
\Pi_{c}(q, \omega)=\frac{9}{4}\left|g(q)\left(1-e^{i q s}\right)\right|^{2}(s / c)\left(\frac{c}{\alpha \pi T}\right)^{2-1 / \theta}\left(1+\frac{s^{2}}{\alpha^{2}}\right)^{1 / 4 \theta-\theta} \times I_{\theta}\left(\frac{\omega-\left(q-2 k_{F}\right) c}{2 \pi T}\right) I_{\theta}\left(\frac{\omega+\left(q-2 k_{F}\right) c}{2 \pi T}\right),
$$

where

$$
I_{\theta}(k)=\frac{1}{2 \pi} \int_{0}^{\infty} e^{i k x}(\sinh x)^{-1 / 2 \theta} .
$$

For $\theta>\frac{1}{2}$ the small $|x \pm c t|$ region does not contribute significantly to the integrals and expression Eq.
(A21) is sufficient. We should remark that for $\theta=\frac{1}{2}$ (the noninteracting fermions, $X Y$ limit) the more exact form of the phase factor must be retained, and Eq. (A22) with $\theta=\frac{1}{2}$ (and multiplied by $\frac{4}{9}$ since there are now no $z$ spin terms) is not the correct form. The final result for the Heisenberg chain is given by $\theta=1$, and is quoted in the text. 


\section{APPENDIX B}

In this Appendix we develop the Ginzburg-Landau expansion to arbitrary order, and derive the zerotemperature results quoted in Sec. V.

The real energy gap $\Delta$ in the excitation spectrum at zero temperature is identified as determining the long-distance behavior of correlation functions through the (long) characteristic length ${ }^{39} v_{F} / \Delta$ (cf. at higher temperatures the temperature provides the infra-red cutoff and determines the characteristic correlation length $v_{F} / T$ ). This is calculated by developing the correlation function as a perturbation expansion in the lattice displacement, and using scaling (homogeneity) arguments (as done by Luther and $P$ eschel ${ }^{8}$ in a different context). Since the free energy (energy at zero temperature) is given by

$$
F=\int_{0}^{Q} d Q^{\prime} \int_{0}^{Q^{\prime}} d Q^{\prime \prime} \Pi_{Q^{\prime \prime}}
$$

where $\Pi_{Q}$ is the linear-response function to a static increment in $Q$, it is convenient to develop the perturbation expansion for $\Pi_{Q}$. This is given by the correlation function Eq. (4.3) but now with averages taken in the distorted state. The expansion is given by going to the interaction representation for any operator $X$

$$
\left\langle X^{\prime}(t) X(0)>_{Q}=<X_{Q}(t) X_{Q}(0)>\right.
$$

where

$$
X_{Q}(t)=U^{\dagger}(t) X(t) U(t)
$$

and

$$
U(t)=T \cdot \exp \left(-i \int_{-\infty}^{t} d t^{\prime} g(q)\left(1-e^{i q s}\right) Q(q) \frac{1}{\sqrt{N}} \sum_{l} e^{i q l s} \overrightarrow{\mathrm{S}}_{l}\left(t^{\prime}\right) \cdot \overrightarrow{\mathrm{S}}_{l+1}\left(t^{\prime}\right)\right)
$$

with $T$ the time-ordering operator. The averages and time dependences after these substitutions are taken in the undistorted state. Developing $U(t)$ in a power series gives the perturbation series in $Q$. Although a detailed evaluation of such a series for the rather general correlation function is clearly impractical, using the general expression for the form of the expectation of an arbitrary product of pseudofermion operators at different points found in Appendix A and then scaling out the variables from the integrals involved, it is rather straightforward to calculate the leading dependence. This is easiest for the terms involving only transverse components of the spin, but we have also shown terms from the $z$ components lead to the same form. Thus at $T=0$ we find

$$
\Pi_{Q}\left(2 k_{F}+\delta q, \omega=0\right) \sim \frac{g^{2}}{J}\left(\frac{1}{s \delta q}\right)^{2-1 / \theta} \sum_{n=0}^{\infty} c_{n}\left[\frac{\Delta_{o}}{J}\left(\frac{1}{s \delta q}\right)^{2-1 / 2 \theta}\right]^{2 n}
$$

where $c_{n}$ are numbers, and we have not distinguished between $s$ and $\alpha$. Requiring the long-wavelength dependence to be of the form

$$
\Pi_{Q}\left(2 k_{F}+\delta q, \omega=0\right) \sim\left(\frac{1}{s \delta q}\right)^{2-1 / \theta} f(\xi \delta q)
$$

with $\xi=v_{F} / \Delta$ (the form of the factor multiplying $f$ comes from the known divergence for $\Delta=0$ ), and comparing with the series expansion leads to the identification

$$
\frac{\Delta}{J} \sim\left(\frac{\Delta_{o}}{J}\right)^{1 /(2-\theta / 2)}
$$

The value $\theta=1$ gives $\Delta \sim J^{1 / 3} \Delta_{o}^{2 / 3}$. Rewriting Eq. (B4) in the form

$$
\Pi_{Q}\left(2 k_{F}+\delta q\right)-\frac{g^{2}}{J}\left(\frac{\Delta_{o}}{J}\right)^{4 \theta /(4 \theta-1)-2} f^{\prime}(\xi \delta q)
$$

and taking the $\delta q \rightarrow 0$ limit gives

$$
\Pi_{Q}\left(2 k_{F}\right) \sim \frac{g^{2}}{J}\left(\frac{\Delta_{o}}{J}\right)^{4 \theta /(4 \theta-1)-2}
$$


where the constant $f^{\prime}(0)$ has been absorbed in the proportionality constant. Equation (B7) then gives for the zero-temperature energy of dimerization of the spin system

$$
\Delta E \sim N J\left(\frac{\Delta_{o}}{J}\right)^{4 \theta /(4 \theta-1)}
$$

which reduces to $E \sim N J^{-1 / 3} \Delta_{o}^{4 / 3}$ for $\theta=1$. Remember $\Delta_{o}$ is proportional to the lattice distortion $Q$.

Note that $\theta=\frac{1}{2}$ (noninteracting fermions) is a special case. Then the first term of the series Eq. (B3) is not of the form written, but instead contains logarithmic corrections, and must be treated separately.

The form of the finite-temperature, $2 k_{F}$ polarizability is given simply by replacing $\delta q$ in Eq. (B3) with $T / c$.

Equation (B1) can then be used to develop the Ginzburg-Landau expansion to arbitrary order.

"Summer visitor; permanent address: Department of Physics, Harvard University, Cambridge, Mass. 02138.

1J. W. Bray, H. R. Hart, Jr., L. V. Interrante, I. S. Jacobs, J. S. Kasper, G. D. Watkins, S. H. Wee, and J. C. Bonner, Phys. Rev. Lett. 35, 744 (1975).

${ }^{2}$ I. S. Jacobs, J. W. Bray, H. R. Hart, Jr., L. V. Interrante, J. S. Kasper, G. D. Watkins, D. E. Prober, and J. C. Bonner, Phys. Rev. B 14, 3036 (1976).

${ }^{3}$ E. Pytte, Phys. Rev. B 10, 4637 (1974).

${ }^{4}$ D. E. Moncton, R. J. Birgeneau, L. V. Interrante, and F. Wudl, Phys. Rev. Lett. 39, 507 (1977).

${ }^{5}$ To avoid confusion we will use "mean-field theory" to refer to the mean-field treatment of the phonons, and "Hartree" (which is also a mean-field theory) to refer to the treatment of the pseudofermions.

6J. M. Luttinger, J. Math. Phys. 4, 1154 (1963).

${ }^{7}$ D. C. Mattis and E. H. Lieb, J. Math. Phys. 6 , 304 (1965).

${ }^{8}$ A. Luther and I. Peschel, Phys. Rev. B 12, 3908 (1975).

9J. C. Bonner and M. E. Fisher, Phys. Rev. A 135 , A610 (1964).

${ }^{10}$ T. D. Schultz, D. C. Mattis, and E. H. Lieb, Rev. Mod. Phys. 36, 856 (1964).

${ }^{11}$ L. N. Bulaevskii, Zh. Eksp. Theor. Fiz. 43, 968 (1962), [Sov. Phys.-JETP 16, 685 (1963)].

${ }^{12}$ The Hartree treatment of the dimerized Heisenberg chain (a) L. N. Bulaevskii (Zh. Eksp. Teor. Fiz. 44, 1008 (1963) [Sov. Phys.-JETP 17, 684 (1963)] ] and by (b) G. Beni [J. Chem. Phys. 58, $3200(1973)]$ is a quite different approximation. In their work a canonical transformation $a_{k} \rightarrow \alpha_{k}$ is performed to diagonalize the $X Y$ part of the Hamiltonian including the phonon coupling, and a Hartree factorization of the new four-fermion $\left(\alpha_{k}\right)$ terms is done. Thus "anomalous averages" $\left\langle a_{k}^{\dagger} a_{k-2 k_{F}}\right\rangle$ are kept in an amount proportional to $g Q$. This, contrary to Pytte's remarks, leads to a quite different estimate of the transition temperature proportional to $\exp \left(-1 / \lambda^{1 / 2}\right)$ in the weakcoupling limit. This second Hartree approach looks dangerous, enhancing the transition temperature using an approximation that if done truly consistently would lead to a nonzero transition temperature even in the absence of phonon coupling - a result known to be wrong.

${ }^{13}$ H. Frohlich, Proc. R. Soc. A 223, 226 (1954).

${ }^{14}$ V. L. Ginzburg, Fiz. Tverd. Tela. 2, 2031 (1960) [Sov. Phys.-Solid State 2, 1824].

${ }^{15}$ Yu. A. Bychkov, L. P. Gorkov, and I. E. Dzyaloshinskii, Zh. Eksp. Theor. Fiz. 50, 738 (1966) [Sov. Phys.-JETP $\underline{23}, 489(1966)]$.
${ }^{16}$ S. T. Chui, T. M. Rice, and C. M. Varma, Solid State. Commun. 15, 155 (1974).

${ }^{17}$ See for example Ref. 16.

${ }^{18}$ T. Wei, A. J. Heeger, M. B. Salamon, and G. E. Delker, Solid State. Commun. 21, 595 (1977).

${ }^{19}$ J. Solyom, J. Low Temp. Phys. 12, 547 (1973).

${ }^{20}$ A. Luther and I. Peschel, Phys. Rev. B 9, 2911 (1974).

${ }^{21} \mathrm{~J}$. des Cloiseaux and J. J. Pearson, Phys. Rev. 128, 2131 (1962).

${ }^{22}$ E. Leib, T. Schultz and D. Mattis, Ann. Phys. 16, 407 (1961).

${ }^{23}$ A. Luther and V. J. Emery, Phys. Rev. Lett. 33,589 (1974).

${ }^{24} \mathrm{The}$ result is quite the contrary for the phonon mediated interaction where we have already shown the umklapp process, [Fig. 2(d)], is much larger than the "exchange" diagram, [Fig. 1(c)], due to the important momentum dependence of the phonon Green's function.

${ }^{25}$ For ease of notation we again imagine one chain, and leave out $\overrightarrow{\mathrm{q}}_{1}$.

${ }^{26}$ R. J. Baxter, Phys. Rev. Lett. 26, 832 (1971); Ann. Phys. (N.Y.) 70, 193 (1972)

${ }^{27}$ The pre-exponential factor is different from that quoted in Refs. $1-3$. It arises from the integral

$\int_{0}^{E_{\max }} d E\left[1-\left(\frac{E}{E_{\max }}\right)^{2}\right]^{1 / 2} \frac{1}{2 E}$

$$
\times \tanh \frac{1}{2} \beta_{c} E=\ln \left(\frac{2}{e} \times 1.13 \beta_{c} E_{\max }\right) .
$$

${ }^{28}$ The good agreement of the magnetic susceptibility with theory described in Refs. 1 and 2 is somewhat mystifying. The theory they use is apparently a mixture taking the order parameter from Pytte's theory (Ref. 3) then using the susceptibility calculation of Bulaevskii (Ref. 13). This we believe is inconsistent. Either theory done consistently would lead to a smaller $\eta$ and worse agreement with experiment.

${ }^{29}$ D. E. Moncton (private communication).

${ }^{30}$ M. C. Leung, Solid State. Commun. 15, 879 (1974).

${ }^{31}$ J. W. Bray, Solid State. Commun.. (to be published)

${ }^{32}$ H. P. van de Braak, W. J. Caspers, F. W. Wiegel, and M. W. M. Willemse (unpublished).

${ }^{33}$ W. Duffy, Jr. and K. P. Barr, Phys. Rev. 165, 647 (1968).

${ }^{34}$ Some care has to be exercised in calculating correlation functions because we have shifted the bands in $k$-space for the antiferromagnetic case. This can result in some spuri- 
ous phase factors in correlation functions. However for the ones of interest these factors cancel.

${ }^{35}$ D. C. Mattis, J. Math. Phys. 15, 609 (1974).

${ }^{36}$ Except that $O_{1}$ and $O_{2}$ as defined here commute instead of anticommuting. It can be shown however (e.g., using the slightly different form of $O_{1}$ and $O_{2}$ of Ref. 35 that preserves the anticommutation) that for all nonzero correlation functions this problem is trivially taken care of by including an explicit phase factor $(-1)^{P_{12}}$, where $P_{12}$ is the number of interchanges of 1 and 2 operators to group all 2 operators together.

${ }^{37}$ A. Luther, Phys. Rev. B 14, 2153 (1976).

${ }^{38} \mathrm{~T}$ he exact phase factor is products of terms of the form $\left[\alpha-i\left(x_{i}-x_{j}\right)\right]^{1 / 2}\left[\alpha+i\left(x_{i}-x_{j}\right)\right]^{-1 / 2}$.

${ }^{39}$ A. I. Larkin and J. Sak, Phys. Rev. Lett. 39, 1025 (1977). 